\title{
Long Term Implications of Climate Change on Crop Planning
}

\author{
Andrew Lewis ${ }^{1}$, Marcus Randall ${ }^{2,1}$, Sean Elliott ${ }^{2}$, and James Montgomery ${ }^{3}$ \\ 1 School of Information and Communication Technology, Institute for Integrated and \\ Intelligent Systems, Griffith University, Queensland, Australia, \\ \{a.lewis,m.randall\}@griffith.edu.au \\ 2 Bond Business School, Bond University, Robina, Queensland, Australia, \\ \{mrandall, selliott\}@bond.edu.au \\ 3 School of Technology, Environments and Design, University of Tasmania, Hobart, \\ Tasmania, Australia, james.montgomery@utas.edu.au
}

\begin{abstract}
The effects of climate change have been much speculated on in the past few years. Consequently, there has been intense interest in one of its key issues of food security into the future. This is particularly so given population increase, urban encroachment on arable land, and the degradation of the land itself. Recently, work has been done on predicting precipitation and temperature for the next few decades as well as developing optimisation models for crop planning. Combining these together, this paper examines the effects of climate change on a large food producing region in Australia, the Murrumbidgee Irrigation Area. For time periods between 1991 and 2071 for dry, average and wet years, an analysis is made about the way that crop mixes will need to change to adapt for the effects of climate change. It is found that sustainable crop choices will change into the future, and that large-scale irrigated agriculture may become unviable in the region in all but the wettest years.
\end{abstract}

Keywords: crop planning $\cdot$ climate change $\cdot$ water management $\cdot$ optimisation $\cdot$ differential evolution.

\section{Introduction}

Climate change is having a large impact on all aspects on animal and plant life across the planet $[11,14,16]$. Emissions and other anthropomorphic effects have seen large changes in political positions and industrial practices [6]. One such industry that is more susceptible to the effects of climate charge, and has a significant impact on the human condition, is agriculture [17]. Given an amount of arable land and a set of climate conditions, the question becomes what may sensibly be planted and how will this change over time? To address this, one particular food growing region in Australia, the Murrumbidgee Irrigation Area (MIA), is examined with a set of climate models, and an optimisation approach tailored towards crop planning. 
The tool used to derive which crop should be planted, and the areas in which to do this, is Differential Evolution (DE) [12,13]. This has been found to be an effective tool for this problem by Montgomery, Fitzgerald, Randall and Lewis [9]. DE uses recombination and mutation to transform vectors so that they improve over time. This is done by gradually reducing the scale of changes made by the algorithm. At the beginning of the search, where the vectors are usually highly distributed, there is scope for poor solutions to make significant improvements in quality as they move towards their better counterparts. As time passes, and vectors become closer and improve. The self-scaling will mean smaller, finer-grained improvements are made. This behaviour helps the population to converge on a good region of state space. In its multi-objective form, DE's standard solution comparison and replacement mechanism (in which a new solution is compared against a single 'parent') is typically replaced by non-dominated sorting over the union of the current population and current iterations' candidate solutions produced using DE's mutation mechanism. While this works against overall population convergence, multi-objective DEs have been effective (see, e.g., Montgomery, Randall and Lewis [10]).

The remainder of this paper is organised as follows. Section 2 describes the use of optimisation tools in crop planning, particularly the recent modelling work that has been done for the MIA. Section 3 explains the problem model and describes the DE implementation. Section 4 examines climate change models, especially NARCLiM that develops predictive models for New South Wales (NSW) in Australia (thus incorporating the area of interest). This section also discusses the processes necessary to handle this data and convert it to a form that is usable by the optimisation model. Section 5 puts all of this together and applies the required computing to determine workable crop mixes in the decades to come. Comment is made on the changes observed. Finally, Section 6 concludes and describes other projects that are now possible because of this work.

\section{The Optimisation of Crop Planning}

The research area of crop planning has seen the application of various optimisation techniques with the aim of generating optimal crop mixes under different climate-related scenarios that maximise revenue while minimising water use. There is a very wide range of research material covering optimisation techniques applied to water management. A good summary of these may be found in Singh [15]. This paper focuses on the water management issues faced by farmers and regional planners in the Murrumbidgee Irrigation Area in south east Australia. The papers that have focused on the MIA have applied a range of computational techniques to generate optimal solutions to the multi-objective problem. The most recent research [7-9] has applied the optimisation techniques Non-dominated Sorting Genetic Algorithm (NSGA-II) and DE to generate good attainment surfaces.

The multi-criteria problem forming the underlying basis of this paper was given initially by Xevi and Khan [18]. Lewis and Randall [7] redefined this as 
a multi-objective problem in their work and applied the NSGA-II optimisation technique to generate the Pareto-efficient solutions to the problem. This work optimised the results of two key output variables. These were to maximise the net revenue generated by the chosen combination of crops and to minimise the corresponding environmental flow deficit. This work also added cotton to the range of crops considered when solving the multi-objective problem. This work was further extended by Montgomery et al. [9]. They considered the application of $\mathrm{DE}$ as the optimisation technique. One of their findings was that the attainment surfaces generated by DE tended to improve on those by the NSGA-II approach.

The previous work considered only current climate conditions. Effectively, the water requirements of each crop have been based on static underlying assumptions relating to temperature and rainfall, where only potential seasonal variation has been incorporated. The purpose of this paper is to project forward in time, on the basis of potential changes to future temperatures and rainfall. This introduces the application of climate change models to show potential future changes to temperatures and rainfall in the MIA. This will make allowance for potential future changes in the water requirement for each crop, and the aim is to generate robust optimal solutions that can withstand potential changes in climate conditions.

\section{Problem Definition and Optimisation}

In the following subsections the problem model and the DE solver used to optimise it are described.

\subsection{Problem Model}

As previously stated, the problem seeks to determine appropriate areas of land for a selection of crops such that a net revenue function is maximised, but an environmental water deficit is minimised. This model is for one year. In the instances used here, the crops that can be selected from are: rice, wheat, barley, maize, canola, oats, soybean, Winter pasture, Summer pasture, lucerne, vines, Winter vegetable, Summer vegetables, citrus, stone fruit and cotton. The multiobjective model used in this paper, reproduced from Lewis and Randall [7] (pp. 181-182) is given in Equations 1-7.

$$
\text { Maximise } \begin{aligned}
N R= & \sum_{c=1}^{C} T C I(c) \times X(c) \\
& -C_{w} \times \sum_{m=1}^{M}\left(\left(\sum_{c=1}^{C} W R E Q(c, m) \times X(c)\right)-P(m)\right) \\
& -C_{p} \times \sum_{m=1}^{M} P(m) \\
& -\sum_{c=1}^{C} V \operatorname{cost}(c) \times X(c)
\end{aligned}
$$


Minimise $E F D=\sum_{m=1}^{M}\left(\right.$ Tenv $\left._{-} f(m)-E n v_{-} f(m)\right) \cdot\left[E n v_{-} f(m)<T e n v_{-} f(m)\right]$

s.t.

$$
\begin{gathered}
\sum_{m=1}^{M} P(m) \leq 50 \mathrm{GL} \\
\sum_{c=1}^{C} X(c) \leq T_{\text {Area }} \\
X(c) \leq Y(c) \quad 1 \leq c \leq C \\
\text { Allocation }(m)=\operatorname{Inflow}(m)-\text { Env_f }(m) \\
P(m)=\left(\sum_{c=1}^{C} W R E Q(c, m) \times X(c)\right)-\text { Allocation }(m)
\end{gathered}
$$

Where:

$N R$ is the net revenue,

$C$ is the number of crops,

$T C I(c)$ is the total crop income for crop $c$,

$X(c)$ is a decision variable which is the area of crop $c$ (in hectares),

$C_{w}$ is total cost of water per unit volume $\$ / \mathrm{ML}$,

$M$ is the number of months, i.e., 12 ,

$W R E Q(c, m)$ is the water required for crop $c$ in month $m$ (in ML),

$P(m)$ is the groundwater pumped in month $m$,

$C_{p}$ is the cost of groundwater pumping and delivery (in $\$ / \mathrm{ML}$ ),

$V \operatorname{cost}(c)$ are all other variable costs associated with crop $c$,

$E F D$ is the deficit in environmental flow, ${ }^{4}$

Tenv $f(m)$ is the target environmental flow for month $m$,

$E n v_{-} f(m)$ is a decision variable that is the environmental flow for month $m$,

$T_{\text {Area }}$ is the total cropping area available,

$Y(c)$ is a the maximum allowable area for crop $c$,

Allocation $(m)$ is the amount of surface water available for irrigation of crops in month $m$ and

Inflow $(m)$ is the total surface (river) water available in month $m$.

\footnotetext{
${ }^{4}$ Equation 2 is expressed in Iverson bracket notation.
} 


\subsection{The DE Solver}

As solutions to this problem are pseudo-continuous it may be solved using continuous solvers operating on integer-valued solution vectors. Differential Evolution (DE) [13], an exemplar continuous solver, has previously been shown to be effective [9] so is used again here. It is adapted to the multiobjective setting using a similar approach to Montgomery, Randall and Lewis in their DE for RFID antenna design [10]: a DE/rand/1/bin algorithm is used to generate new solutions from the current population, after which the next generation is selected by applying the non-dominated sorting algorithm from NSGA-II [3] to the union of these solution sets. Feasible solutions are compared using standard Paretodominance rules, while a feasible solution dominates any infeasible solution, and infeasible solutions are compared based on the amount they violate the pumped water constraint (to provide some selection pressure toward feasible space).

The population (hence, archive) size is set to 100 members, and the algorithm is executed for 10,000 iterations (one million function evaluations). Appropriate values of difference vector scale $F$ and crossover probability $C r$ are 0.8 and 0.5 , respectively.

Solutions are represented as vectors of $C+12$ integer values (i.e., 28 in the current problem as there are 16 crops) corresponding to the areas allocated to the $C$ crops and environmental flows for each month of the year. Certain highly lucrative crops (vines, summer and winter vegetables, citrus, and stone fruit) have restrictive upper bounds on their areas, set at $10 \%$ of Australian national production. The ranges of the remaining 'unbounded' crops are 0 to the size of the farming land (120,000 ha), while the bounded crops are restricted to 0 to their nominated maximum. Further details of these values may be found in Lewis and Randall [7]. Environmental flow variables range between 0 and the total inflow for their respective months (which will often exceed the target environmental flow, although in some months they are less than the target).

Initial solutions for the $\mathrm{DE}$ are created by the following steps:

1. Generate $C$ uniform random values $r_{c}$ in $[0,1]$.

2. Allocate each bounded crop $c$ with $r_{c} \geq 0.5$ its maximum area.

3. Normalise the $r_{c}$ values for all remaining crops with $r_{c} \geq 0.5$, then allocate those crops space from the remaining area in proportion to their normalised $r_{c}$ values.

4. Generate 12 randomised integer values in the range $[0, \operatorname{Inflow}(m)]$ for each month $m$ to set the solution's environmental flows.

\section{Using Climate Predictions to Inform Crop Planning Models}

As may be noted, in order to model climate impact on optimal crop selections, some knowledge of crop water requirements and available water sources are needed. Our previous work made use of published data (rainfall, reference evapotranspiration, and surface water supplies - streamflows in rivers used to supply 
water for irrigation) for typical years (wet, dry and average) for the MIA. This data had been accumulated from meteorological observations over a number of years, but the specific sources were not supplied. In any case, data for future years cannot be sourced from historical observations.

In order to compile the required data, recourse was made to the NSW and ACT Regional Climate Modelling (NARCliM) Project $[4,5]$. This is a research partnership between the NSW and ACT state governments in Australia and the Climate Change Research Centre at the University of NSW. It has generated projections from four global climate models dynamically downscaled by three regional climate models, for three time periods: 1990 to 2009 (base), 2020 to 2039 (near future), and 2060 to 2079 (far future). Meteorological data are available on a $10 \mathrm{~km}$ grid across the NARCliM domain, which covers most of S.E. Australia.

For the purposes of the work described in this paper, data were extracted from the R3 physical configuration of the Weather Research and Forecasting model downscaling of the CCCMA 3.1 global climate model. This combination was chosen for this preliminary investigation because its results lie closest to the mean of the 12 model ensemble generated by the NARCliM project. Use of data from other models is planned for future work investigating the extremes of climate predictions, and uncertainties of future climate outcomes.

Data were extracted from the CCCMA3.1-R3 output data sets covering a 120,000 ha region north and west of the approximate location of the Berembed Weir on the Murrumbidgee River. This approximates the area of the MIA modelled in previous work. The data used were monthly averages of mean, maximum and minimum daily temperatures, and precipitation. The data were aggregated to give monthly mean values over the entire region.

In addition, data were aggregated over an 800,000 ha area covering the Snowy Mountains region. Precipitation data were averaged over this region to give an approximation to the available water sources in the catchment for the Murrumbidgee River.

From inspection of the annual aggregate precipitation data for the MIA, three years were selected from each modelled time period to represent wet, dry and average years. "Wet" years and "dry" years were those with the greatest and least aggregate annual rainfall in the relevant period, respectively. Apart from these two, obvious extremes, the "average" years were chosen as those with an aggregate annual rainfall closest to the 20 year mean annual rainfall. The chosen years are shown in Table 1.

\begin{tabular}{|c|c|c|}
\hline Wet & Averag & Dry \\
\hline$\overline{1991}$ & 2002 & 200 \\
\hline 2038 & 2037 & 20 \\
\hline 063 & 2071 & \\
\hline
\end{tabular}

Table 1. Representative years selected for modelling 
From each of the selected years, average minimum and maximum daily temperatures were used to calculate reference evapotranspiration data by way of a temperature-based application of the FAO Penman-Monteith equation [1]. The calculated monthly $E T_{0}$ are shown in Table 2 . As previously noted, monthly average rainfall was aggregated over the area to be modelled. These data are shown in Table 3.

\begin{tabular}{ll|llllllllllll} 
& & & Jan & Feb & Mar & Apr May & Jun & Jul & Aug & Sep & Oct & Nov & Dec \\
\hline \multirow{4}{*}{ Wet } & 1991 & 1.96 & 1.53 & 1.27 & 0.81 & 0.55 & 0.38 & 0.43 & 0.54 & 0.80 & 1.23 & 1.53 & 1.93 \\
& 2038 & 1.92 & 1.48 & 1.37 & 0.89 & 0.57 & 0.39 & 0.42 & 0.56 & 0.78 & 1.31 & 1.73 & 1.93 \\
\multirow{4}{*}{ Average 2002 } & 1.93 & 1.47 & 1.45 & 0.85 & 0.57 & 0.43 & 0.44 & 0.51 & 0.86 & 1.43 & 1.61 & 1.99 \\
& 2063 & 2.17 & 1.46 & 1.39 & 0.88 & 0.61 & 0.40 & 0.41 & 0.56 & 0.77 & 1.30 & 1.83 & 2.05 \\
\multirow{4}{*}{ Dry } & 2037 & 2.05 & 1.39 & 1.35 & 0.95 & 0.59 & 0.41 & 0.42 & 0.59 & 0.87 & 1.39 & 1.84 & 2.00 \\
& 2071 & 2.21 & 1.80 & 1.55 & 0.94 & 0.60 & 0.42 & 0.45 & 0.63 & 0.84 & 1.45 & 1.78 & 1.99 \\
& 2003 & 2.03 & 1.83 & 1.47 & 1.05 & 0.78 & 0.43 & 0.43 & 0.63 & 0.94 & 1.65 & 2.03 & 2.05 \\
& 2033 & 1.90 & 1.54 & 1.48 & 0.95 & 0.65 & 0.48 & 0.44 & 0.66 & 1.03 & 1.42 & 1.90 & 1.97 \\
& 2064 & 2.03 & 1.74 & 1.45 & 1.03 & 0.75 & 0.40 & 0.46 & 0.69 & 0.89 & 1.53 & 1.97 & 2.03
\end{tabular}

Table 2. Reference Evapotranspiration $\left(E T_{0}\right)$

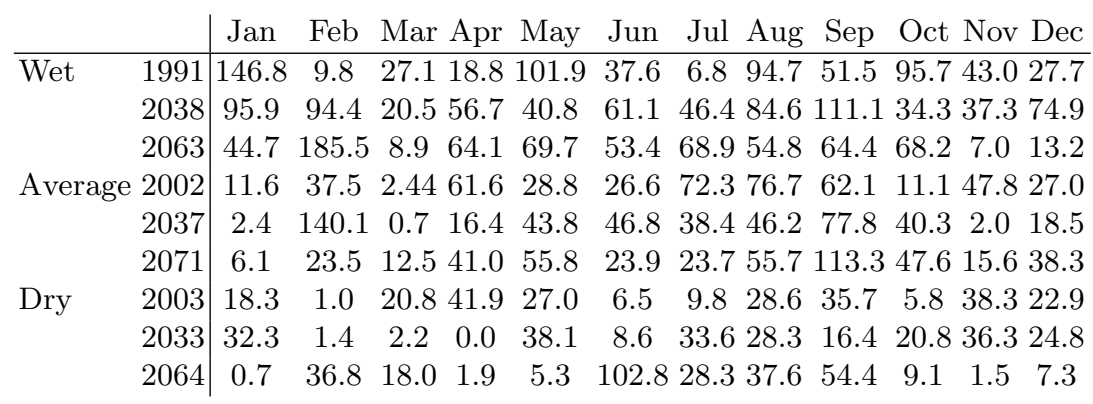

Table 3. Average monthly precipitation for MIA (mm)

Finally, it was necessary to calculate some approximation to the streamflow in the Murrumbidgee River, to provide estimates of surface water available for irrigation. Limited data were available for Berembed Weir directly, for the baseline years (1990 to 2009), but a more comprehensive series of data was available for the Murrumbidgee River at Wagga Wagga (Station 410001, Lat: -35.10, Long: 147.37, NSW Department of Industry - Lands and Water [2]) As there is no major intervention in streamflow between the Wagga Wagga station and the 
main canal off-take at Berembed Weir, streamflow data at Wagga Wagga was taken as a reasonable proxy for water available for irrigation at the weir. This was adequate for the years selected in the baseline, for which there were data available in the historical record, but more problematic for future years, in the timeframes of the climate predicted data. A median ratio was determined between the aggregate monthly precipitation in the Snowy Mountains catchment area, and the measured streamflow at Wagga Wagga, for the 20 years of the baseline. This can only be a gross approximation to actual streamflow, which is often determined by human intervention (water releases from Burrinjuck Dam on the Murrumbidgee River and Blowering Dam on the Tumut River). The median ratio was then applied to the aggregate monthly precipitation extracted from the climate model outputs in the Snowy Mountains catchment for the years selected in future time periods. These data are shown in Table 4.

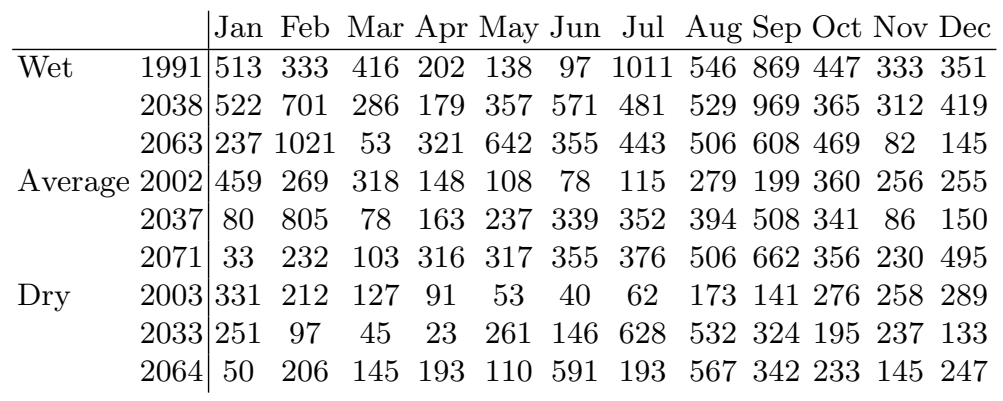

Table 4. Monthly streamflow - Murrumbidgee River at Wagga Wagga (GL)

With this accumulated data, it is possible to calculate the water required, per hectare, for cultivation of a crop, $c$, in a particular month, $m$, from Equation 8:

$$
W R E Q(c, m)=k_{c}(c, m) \times E T_{0}(m)-\operatorname{Rain}(m)
$$

where $k_{c}(c, m)$ is the crop coefficient for that particular point in the crop's growing season, and $E T_{0}(m)$ and $\operatorname{Rain}(m)$ are determined from Tables 2 and 3, respectively. Taken together with the streamflow (surface water available for irrigation) from Table 4, modelling of optimal crop selections can proceed.

\section{Computational Experiments and Results}

The DE solver described above was applied to determine optimal crop selection and environmental flow data, using the model previously described to evaluate the twin objectives of maximum net revenue and minimum "environmental flow deficit", the difference between the monthly environmental flow set as one of a 
trial solution's parameters and a "target", monthly, environmental flow to be released downstream. For the purposes of this preliminary study, an arbitrary monthly target of $100 \mathrm{GL}$ was set for all months. Ten optimisation runs were performed using random seeds for each of the selected years in the three time periods, and median data inspected. The solution sets produced for each instance were highly consistent across trials (yet highly distinct between instances), suggesting that the solver was able to approximate the true Pareto fronts. (For reasons of simplicity and consistency, results reported in this paper are for single runs that closely approach median values.) The results are presented as objective attainment surfaces in following subsections.

Preliminary runs indicated some problems with environmental flow releases in future scenarios. In search of revenue, many trial solutions were generated that reduced environmental flow to zero, i.e., the river downstream was entirely dry. This was obviously considered unreasonable, so an arbitrary constraint was applied to maintain a minimum, monthly environmental flow of 30 GL.

In order to achieve the minimum environmental flows, it was necessary to make alterations to some projected inflows, as in some cases these were below the 30 GL limit. A decision was made to "top up" the monthly inflow to 50 GL whenever it fell below the limit, subtracting a corresponding amount from the following month. This was intended to model dam releases, and the $50 \mathrm{GL}$ figure chosen to supply at least some water for irrigation before the minimum was reached.

As all nine scenarios include months where the inflow is less than the 100 GL target, the minimum achievable environmental deficit is always greater than zero. Across all scenarios and trials the optimisation algorithm was able to find solutions to meet these minima. Therefore, the lower extent of the attainment surfaces is a realistic indication of the extent to which the flow targets can be achieved. Ideally, these targets should be based on environmental cease-flow thresholds for downstream ecosystems, instead of arbitrarily imposed limits, and future work is to be directed towards this.

\subsection{Wet years}

From Figure 1 it may be seen that, in the baseline period, the optimisation algorithm has converged to a single solution. This represents $100 \%$ use of available land, with maximum allowed allocation to vegetable crops and citrus, and the remainder planted with cotton. There was sufficient water to achieve minimal environmental flow deficit and the highest net revenue achieved.

In the near-future scenario, it was no longer possible to maximise net revenue without incurring deficits in environmental flows, though they are relatively small in scale. It was possible to minimise these deficits by reducing cotton cultivation by $30 \%$ and replacing it with canola.

In the far future, net revenue achievable was near halved, compared to baseline, and flow deficits increased. No solutions were capable of using all available land, and to reach minimum flow deficits less than half the land was cultivated. 


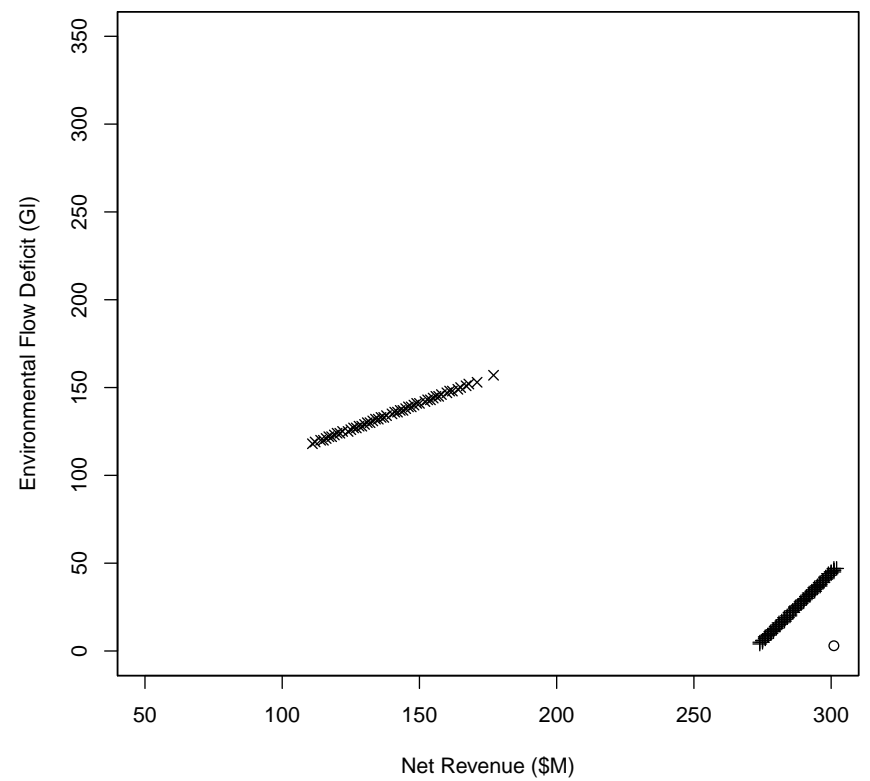

Fig. 1. Objective attainment surfaces for "wet" years, 1991 (o), 2038 (+) and 2063 $(\times)$.

In all solutions it proved infeasible to grow cotton, its place being taken by canola, with maximal crops of vegetables and citrus.

\subsection{Average years}

From Figure 2 it may be seen that there are several similar trends in results for average years, compared to wet years. The baseline year did not have enough water to converge to a single solution, instead spanning from almost as much net revenue but with $100 \mathrm{GL}$ flow deficit, down to minimal flow deficit with $9 \%$ less net revenue. In the near future, net revenues were again nearly halved, and flow deficits increased. To achieve minimal flow deficits, cultivated land had to be severely curtailed. Crop selection still always included maximum vegetables and citrus, with a mix of cotton and canola filling the remainder. In the far future, it was once more infeasible to grow cotton, and again it was replaced by canola. To achieve minimal flow deficits, less than $40 \%$ of available land was cultivated.

\subsection{Dry years}

Results for "dry" years are shown in Figure 3. All years, from each of the periods, show significant environmental flow deficits - there is simply not enough water to 




Fig. 2. Objective attainment surfaces for "average" years, 2002 (o), 2037 (+), and 2071 $(\times)$.

achieve the (arbitrary) 100 GL targets each month in a dry year. In the baseline year, to achieve the maximum net revenue required an aggregate flow deficit of over $300 \mathrm{GL}$ - targets were not reached for two thirds of the year. Flow deficits could be halved, with a corresponding $19 \%$ reduction in net revenue (and land cultivated). Maximum vegetable and citrus crops were included with a varying mix of cotton and canola.

For the near future, similar flow deficits were experienced, but with significantly reduced net revenue - at best $71 \%$ compared to the baseline, reducing to $40 \%$ if flow deficits are minimised. All solutions were unable to use all available land, ranging from a maximum of $85 \%$ down to $40 \%$. Vegetables and citrus remained close to maximums across solutions. The cotton crop remained fairly stable at about 30,000 ha, with canola ranging from a minimum of 10,000 ha to a maximum of 45,000 ha, making an increasing contribution to an increasing net revenue.

In the far future, no cotton crop was possible. Maximum vegetable and citrus crops underpinned net revenue, with an increasing area of canola across solutions as net revenue increased. Net revenue only reached a maximum of one third of that achieved in the baseline years. Less than half the land available was cultivated, reducing to only $17 \%$ if environmental flows were to be maintained. 


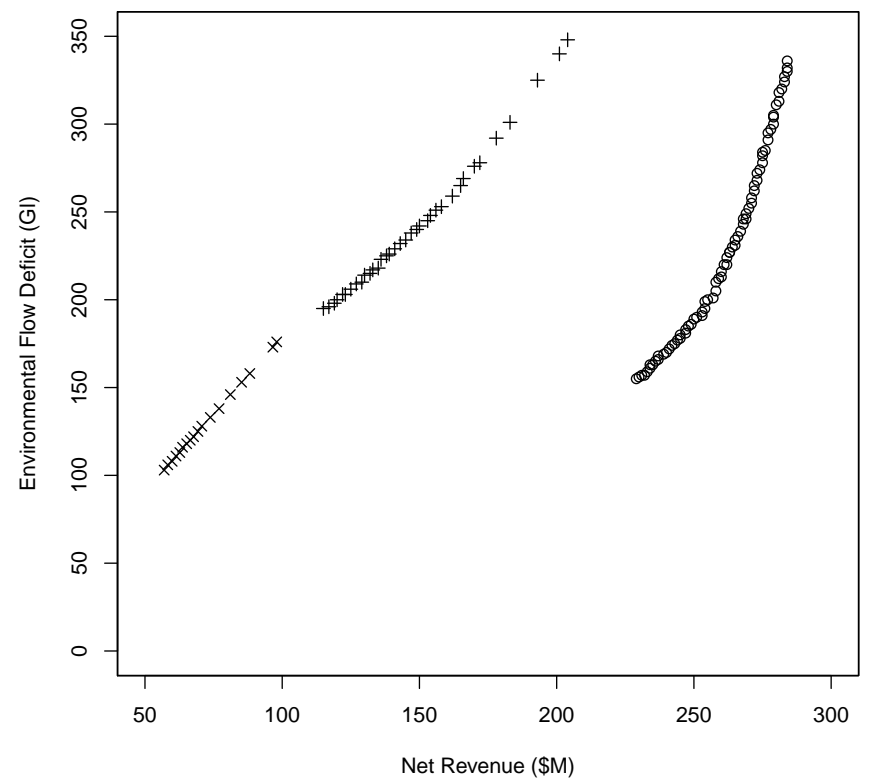

Fig. 3. Objective attainment surfaces for "dry" years, 2003 (o), 2033 (+), and 2064 $(\times)$.

To summarise, net revenues can be seen generally to decline to half, in the near future, or a third or less in the far future. Crops forming the basis for revenues in the baseline, e.g., cotton, become increasingly non-viable as climate changes progress over the near and far future. Inspection of solution details showed that by the far future period, in a wet year the area cultivable decreased to, at best, $90 \%$ of the total if environmental flow requirements were ignored and $50 \%$ if they were taken fully into account. In a dry year, less than $50 \%$ of the total area was cultivable, or less than $20 \%$ if environmental flows were to be maintained.

All solutions included crop allocations that approach the (arbitrary) maximum limits placed on perishable commodities - it is possible that closer consideration of these limits, and the potential influence of population and market demand, might significantly change optimal choices. For this reason the results reported here should not be considered as recommending particular courses of action.

From these preliminary results, it appears that if sufficient water is released for aggregate downstream needs - environmental, agricultural and to support human populations - it may be that large-scale, irrigated agriculture may become unsustainable or uneconomic in the region in all but the wettest years. 
It should be noted that this study only considers the impact of projected water availability on irrigated agriculture. No attempt has yet been made to incorporate changing crop yields as ambient temperatures and humidity change, or other foreseeable impacts on agro-economic and environmental systems.

Furthermore, the discussion of results is centred around characteristic "wet", "average" and "dry" years - there also has been no attempt yet to determine the (possibly changing) proportions of years in different time periods that can be characterised by these different labels. A brief inspection of the predicted climate

data on which the modelling is based shows that by the far future period, $60 \%$ of years have less aggregate annual rainfall that the baseline average for the MIA, and $70 \%$ have less than baseline average precipitation in the Snowy Mountains catchment. Of perhaps greater concern is that the baseline period, 1990-2009, brackets the "Millenium drought" in Australia, recognised as the worst drought on record. For available water for agriculture in the MIA to be predicted to be predominantly less than available during this period is gravely concerning, and deserves further investigation.

\section{Conclusions}

Changes to the world climate will have profound implications for human centred activities, particularly the growing of crops to ensure food security. Using recognised climate models, the research reported in this paper has sought to shed light on some of the potential impacts of, in particular, future water availability on large-scale, irrigated agriculture, using the Murrumbidgee Irrigation Area (MIA) as a case study. It is important to note that the current and future, predicted conditions are specific to the MIA, and any conclusions and observations are solely applicable to this region.

The results obtained are preliminary, with many factors as yet unaccounted for. However, the framework and modelling approach described provide a foundation for future extension and development - it is easy to see how further detail of climate-related impacts, agronomic considerations of soil pathogen control, and commonly employed variations in sowing, crop species and irrigation strategies could be incorporated, to ensure modelling is more realistic and relevant to real-world practice. Collaborative research ties are being pursued to achieve this. The results reported are sufficiently concerning in their implications - that in the future large-scale, irrigated agriculture may become unsustainable in the region - that extensive future work is planned and underway.

The use of time in this paper is consistent with a snapshot approach (i.e., examining different time periods in isolation, and assuming complete knowledge of available water at the start of the season). An ongoing practical consideration for farmers and regional planners, though, is what crops should be grown over a certain timeframe (such as a decade) and how the crop mix should change over that time. To that end, a temporal model is currently being developed which attempts to maximise overall revenue while minimising cumulative water usage over extended timeframes. 


\section{References}

1. Allen, R.G., Pereira, L.S., Raes, D., Smith, M., et al.: Crop evapotranspirationguidelines for computing crop water requirements-FAO irrigation and drainage paper 56. Food and Agriculture Organization, Rome 300(9), D05109 (1998)

2. Bureau of Meteorology (Australia): http://www.bom.gov.au/waterdata/, Data owner: NSW Department of Industry - Lands and Water

3. Deb, K., Pratap, A., Agarwal, S., Meyarivan, T.: A fast and elitist multi objective genetic algorithm: NSGA-II. IEEE Transactions on Evolutionary Computation 6, 182-197 (2002)

4. Evans, J., Ji, F., Lee, C., Smith, P., Argüeso, D., Fita, L.: Design of a regional climate modelling projection ensemble experiment - NARCliM. Geoscientific Model Development 7(2), 621-629 (2014)

5. Evans, J., McCabe, M.: Regional climate simulation over Australia's MurrayDarling Basin: A multi-temporal assessment. Journal of Geophysical Research: Atmospheres 115 (2010). https://doi.org/doi:10.1029/2010JD013816.

6. Kolk, A., Pinkse, J.: Multinationals' political activities on climate change. Business \& Society 46(2), 201-228 (2007)

7. Lewis, A., Randall, M.: Solving multi-objective water management problems using evolutionary computation. Journal of Environmental Management 204, 179-188 (2017)

8. Lewis, A., Randall, M., Capon, S., Jackwitz, E.: Constrained optimisation of agricultural water management with parameter-sensitive objectives. In: Proceedings of the $15^{\text {th }}$ International Conference on Computer Applications. pp. 79-85 (2017)

9. Montgomery, J., Fitzgerald, A., Randall, M., Lewis, A.: A computational comparison of evolutionary algorithms for water resource planning for agricultural and environmental purposes. In: Proceedings of the 2018 IEEE Congress on Evolutionary Computation. pp. 1-8 (2018)

10. Montgomery, J., Randall, M., Lewis, A.: Differential evolution for RFID antenna design: A comparison with ant colony optimisation. In: Genetic and Evolutionary Computation Conference. pp. 673-680. Dublin, Ireland (2011)

11. Parmesan, C., Yohe, G.: A globally coherent fingerprint of climate change impacts across natural systems. Nature 421(6918), 37 (2003)

12. Price, K.: An introduction to differential evolution. In: Corne, D., Dorigo, M., Glover, F. (eds.) New Ideas in Optimization, pp. 79-108. McGraw Hill (1999)

13. Price, K., Storn, R., Lampinen, J.: Differential Evolution: A Practical Approach to Global Optimization. Springer (2005)

14. Rosenzweig, C., Parry, M., et al.: Potential impact of climate change on world food supply. Nature 367(6459), 133-138 (1994)

15. Singh, A.: Review: Computer-based models for managing the water-resource problems of irrigated agriculture. Hydrogeology Journal pp. 1-11 (2015)

16. Stern, N., Peters, S., Bakhshi, V., Bowen, A., Cameron, C., Catovsky, S., Crane, D., Cruickshank, S., Dietz, S., Edmonson, N.: Stern Review: The economics of climate change, vol. 30. HM Treasury London (2006)

17. Turral, H., Burke, J., Faurès, J.: Climate change, water and food security. Food and Agriculture Organization of the United Nations Rome, Italy (2011)

18. Xevi, E., Khan, S.: A multi-objective optimisation approach to water management. Journal of Environmental Management 77(4), 269-277 (2005) 